\title{
TUNGSTEN OXIDE NANOSTRUCTURES FOR ENERGY STORAGE AND FIELD EMISSION APPLICATIONS
}

\author{
Shreyasi Pal ${ }^{1}$, Kalyan Kumar Chattopadhyay ${ }^{2}$ \\ ${ }^{1}$ Senior Research Fellow, Department of Physics, Jadavpur University, West Bengal, India \\ ${ }^{2}$ Professor, Department of Physics, Jadavpur University, West Bengal, India
}

\begin{abstract}
Single crystalline tungsten oxide $\left(\mathrm{WO}_{3}\right)$ nanorods were synthesized over flexible carbon fabric via simple hydrothermal method and used directly as supercapacitor (SC) electrode. $\mathrm{WO}_{3}$ nanorods with their peculiar structural features over the fabric exhibited excellent electrochemical performance with a high specific capacitance of $481.34 \mathrm{~F} / \mathrm{g}$ at scan speed $10 \mathrm{mV} \mathrm{s}{ }^{-1}$. Additionally, these nanorods showed propitious as electron field emitters where the registered turn on field and estimated field enhancement values were $3.6 \mathrm{~V} / \mathrm{\mu m}$ and 4550 respectively. These results indicate that present unique $\mathrm{WO}_{3}$ nanorods arrays over flexible carbon cloth substrate are a promising candidate for constructing high-performance solid state electronics and electrochromic devices.
\end{abstract}

Key Words: Semiconductor compounds, Nanorods, carbon fabric, Supercapacitors, Field emitters and arrays

\section{INTRODUCTION}

From the rich assortment of transition metal oxides, Tungsten oxide $\left(\mathrm{WO}_{3}\right)$, an n-type semiconductor material, has attracted worldwide research interest due to its unique physical and chemical features [1]. Recently, generous amount of efforts have been devoted to synthesize one dimensional (1D) nanostructured $\mathrm{WO}_{3}$ owing to their potential propitious in diverse arena like field emission [2,3], gas sensing [4], photo-catalysis [5] and electrochromic devices [6] where $\mathrm{WO}_{3}$ nanostructured arrays were developed via thermal evaporation [7], chemical vapor deposition [8,9] electrochemical anode oxidation [10], solgel processing [11], hydrothermal approaches and etc. Among these synthesis protocols, hydrothermal technique is very appealing owing to its low temperature processing and environment friendliness. Among the widespread application windows of $\mathrm{WO}_{3}$, its usability as supercapacitor is very interesting from both scientific and technological aspects. Modern era with ever increasing energy demand and rapid exhaustion of the limited global resources made the supercapacitor (SC) a best alternative for environmental benign, budgetary rechargeable power source in foreseeable devices. On the other hand, field emission, field assisted electron emission from these semiconductor nanostructure persistently researched for last decade where the electron emission form the emitter is beneficiated form the peculiar nanometric geometry of the nanostructure. Furthermore, previous documentations on field emission were mainly confined to carbon based inorganic materials and other oxides based semiconductors where works on field electron emission of $\mathrm{WO}_{3}$ is still less cultivated thus requiring attention in full throttle.

In this work, we develop a simple strategy to fabricate $\mathrm{WO}_{3}$ nanorods (NRs) over flexible carbon fabric via facile low temperature hydrothermal method. Choice of carbon fabric as substrate is directed by its enormous advantageous like chemical inertness, excellent thermal stability, nearly metallic conductivity and emission beneficial woven like geometry etc.[12] Additionally, the role of carbon fiber as field electron emission booster has already demonstrated. The as prepared sample was directly used as supercapacitor which bestows decent specific capacitance beneficiary of the binder-free electrode structure and the presence of underlying conducting carbon cloth which induce good conductivity. Being not restricted to electrochemical investigation only, we have also studied their field electron emission performance where the 1D nanostructure on behalf of their arrangement over the carbon cloth and large aspect ratio showed good electron emission characteristics. The underlying conducting carbon cloth with special woven like geometry not only provides the additional geometric enhancement but also fulfills the flexibility requirement.

\section{EXPERIMENTAL}

Chemicals of analytic grade were used for synthesis of $\mathrm{WO}_{3}$ nanorods. Firstly, a sol was prepared by mixing appropriate amount of tungstic acid $\left(\mathrm{H}_{2} \mathrm{WO}_{4}\right)$, polyvinyl alcohol (PVA) and hydrogen peroxide $\left(\mathrm{H}_{2} \mathrm{O}_{2}\right)$. Then, ultrasonically cleaned carbon cloths were dip coated in the sol and dried overnight in regular oven.

\subsection{Preparation of a stock solution}

Initially a $\mathrm{WO}_{3}$ stock solution was prepared by mixing tungstic acid, deionized (D.I.) water and hydrogen peroxide and vigorously stirring the whole mixture at $\sim 95^{\circ} \mathrm{C}$ until a transparent solution was formed and then kept at room temperature. After cooled down to room temperature, the stock solution was diluted with D.I. water.

\subsection{Synthesis of $\mathrm{WO}_{3}$ nanorods}

Now for the synthesis of $\mathrm{WO}_{3} \mathrm{NRs}$, appropriate amount of stock solution was added with hydrochloric acid and acetonitrile and the total solution was transferred to $25 \mathrm{~mL}$ 
autoclave. Thereafter, the seeded carbon cloth substrate was dipped into it and the whole system was kept at $180^{\circ} \mathrm{C}$ for 24 hours. Finally, the substrate was washed with copious amount of D.I. water and dried overnight.

\section{CHARACTERIZATION}

Product morphology was investigated with field emission scanning electron microscope (FESEM, HITACHI S-4800) equipped with an energy dispersive X-ray attachment (EDX) and high resolution transmission electron microscope (HRTEM, JEM 2100). Crystallinity and phase of the synthesized artifacts were characterized by X-ray diffraction (XRD, D8 Advanced Bruker).

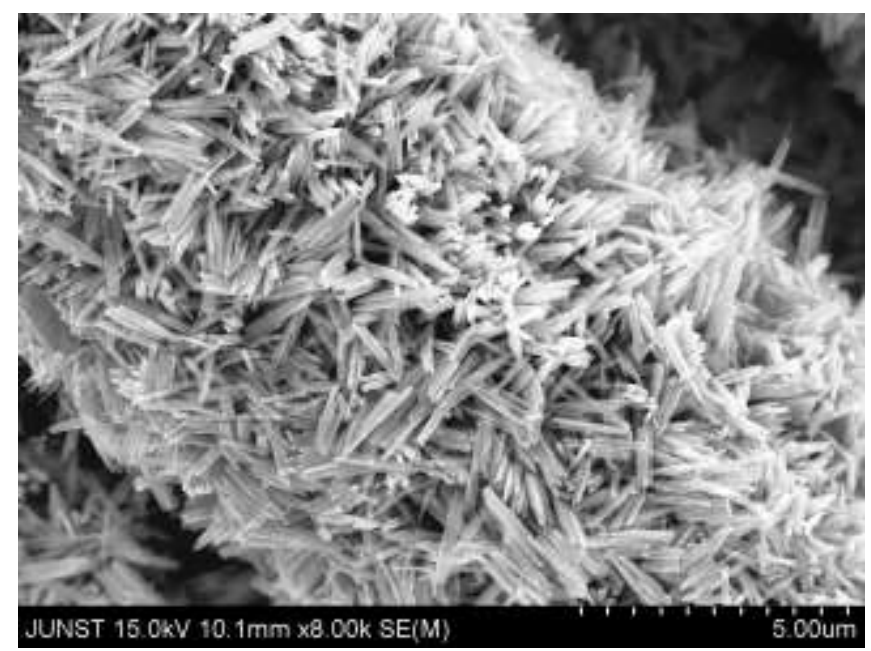

Fig -1: FESEM image of WO3 nanorods

\subsection{Electrochemical measurement}

All electrochemical measurements were carried out with two electrode cell with $\mathrm{PVA}-\mathrm{H}_{3} \mathrm{PO}_{4}$ gel as the electrolyte. $\mathrm{WO}_{3}$ nanorod arrays supported on carbon cloth were used as the working electrode. The $\mathrm{PVA}-\mathrm{H}_{3} \mathrm{PO}_{4}$ gel electrolyte was prepared by adding appropriate amount $\mathrm{H}_{3} \mathrm{PO}_{4}$, D.I. water and PVA and stirring the solution at $85{ }^{\circ} \mathrm{C}$ until the solution became clear. Two pieces of as prepared samples were immersed into the PVA- $\mathrm{H}_{3} \mathrm{PO}_{4}$ gel electrolyte and thereafter they were assembled one by one and kept at room temperature.

\section{Results \& Discussion}

Low magnification FESEM image of $\mathrm{WO}_{3}$ NRs over flexible carbon fiber in Fig.1 reveals large scale morphological uniformity over the substrate. Free standing $\mathrm{WO}_{3}$ NRs grown over the entire cylindrical surface of the individual fiber having radiating tip outwards from the axis of fiber is obvious from this figure. The average diameter and length of NRs are $\sim 50-100 \mathrm{~nm}$ and $\sim 1 \mu \mathrm{m}$ respectively.
High resolution transmission electron microscopy (HRTEM) technique was used further to investigate the crystallinity of the developed $\mathrm{WO}_{3}$ nanostructures. A HRTEM image and corresponding FFT pattern of $\mathrm{WO}_{3} \mathrm{NRs}$ indicate that the nanorods are single crystals with the preferred [002] growth direction (Fig.2).

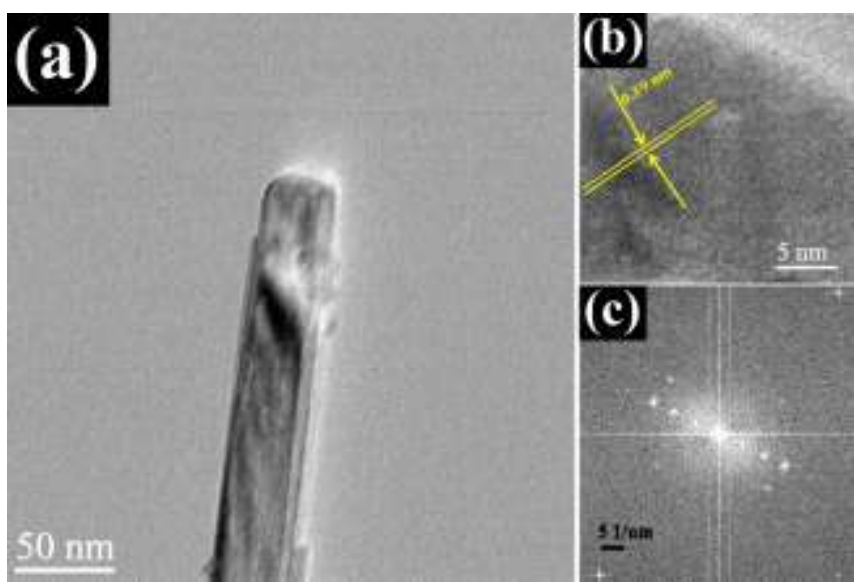

Fig -2: HRTEM image and FFT pattern of $\mathrm{WO}_{3}$ nanorods.

To check phase content of the nanostructure, X-ray diffraction (XRD) analysis was carried out. All the diffraction peaks in this pattern (Fig.3), can be well-indexed to $\mathrm{WO}_{3}$. High intensity of these sharp peaks indicated that the synthesized $\mathrm{WO}_{3}$ nanorods have good crystallinity.

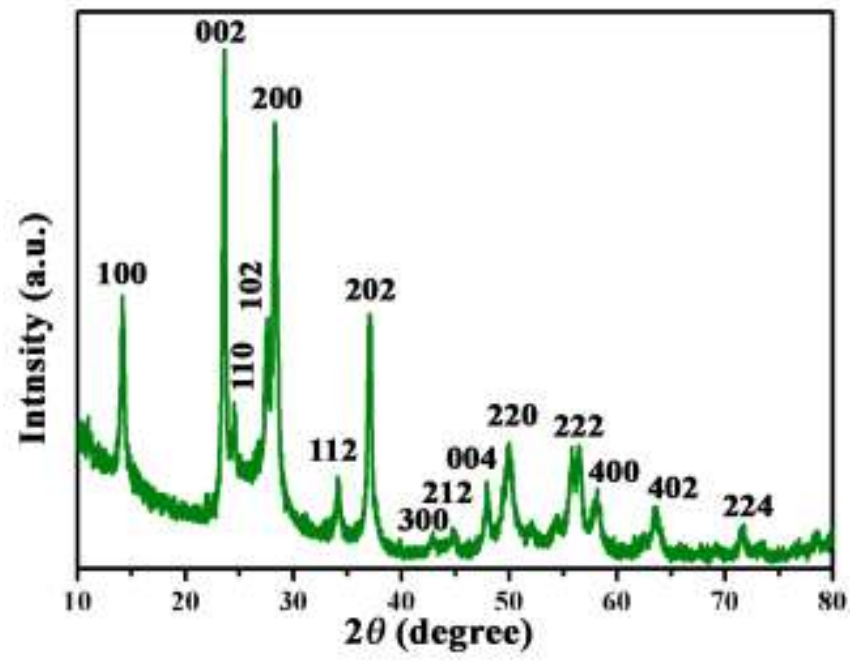

Fig -3: XRD pattern of $\mathrm{WO}_{3}$ nanorods.

EDX analysis and corresponding elemental mapping of the $\mathrm{WO}_{3}$ were also carried out and presented in Fig.4. This EDX mapping images corresponding to $\mathrm{W}$ and $\mathrm{O}$ confirm that the synthesized samples are exclusively composed of $\mathrm{W}$ and $\mathrm{O}$ only. 

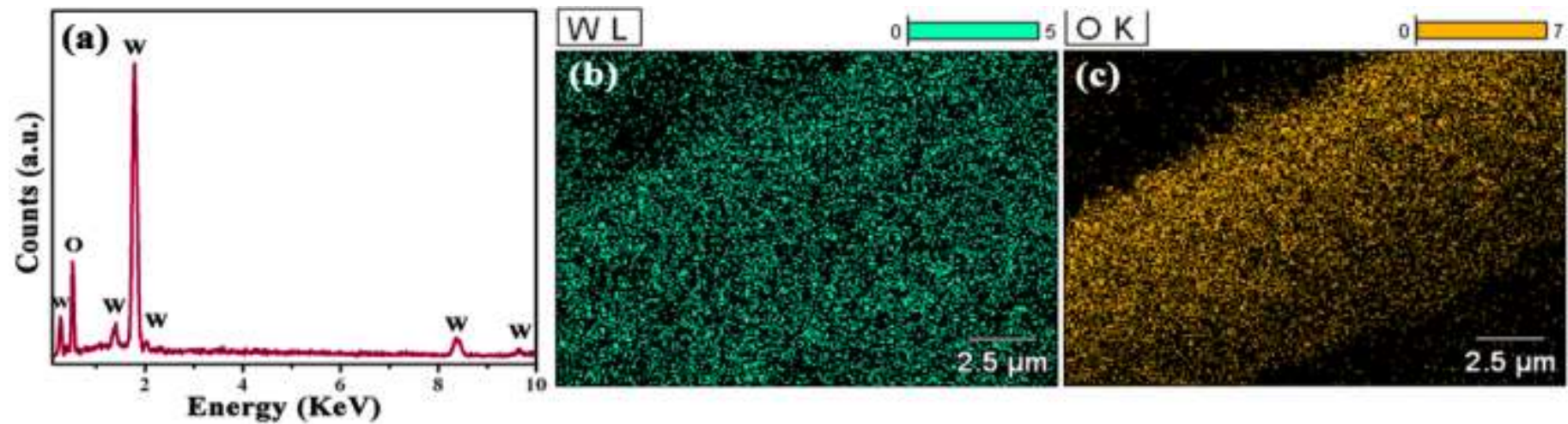

Fig -4: (a) EDX pattern of $\mathrm{WO}_{3}$ nanorods, and (b,c) corresponding elemental mapping showing the homogeneous distribution of the $\mathrm{W}$ and $\mathrm{O}$ throughout the entire nanostructure.

\subsection{Electrochemical measurement}

To explore the potential applications of the as prepared samples in supercapacitors, electrochemical performance of $\mathrm{WO}_{3} \mathrm{NRs}$ as an integrated electrode was carried out.

The electrochemical performances of $\mathrm{WO}_{3} \mathrm{NRs}$ electrodes for supercapacitors are tested by using cyclic voltammetry (CV) curves Fig. 5a compares the CV curves of the $\mathrm{WO}_{3}$ NRs and pure carbon fiber at $20 \mathrm{mV} \mathrm{s}^{-1}$, from which we clearly observe that the integrated electrode exhibits much higher capacitive current density than the bare carbon cloth, thereby discloses that the pure cloth contributes slightly to the total capacitance of the nanostructured electrode. A possible charge storage mechanism taken place on the $\mathrm{WO}_{3}$ electrode surface is shown as follows:

$$
W O_{3}+x H^{+}+x e^{-} \leftrightarrow H_{x} W O_{3}
$$

Further, $\mathrm{CV}$ curves of the $\mathrm{WO}_{3}$ electrode recorded at various scan rates ranging from 10 to $50 \mathrm{mV} \mathrm{s}^{-1}$ are shown in Fig. 5b. The specific capacitances of $\mathrm{WO}_{3} \mathrm{NRs}$ electrode are calculated from the $\mathrm{CV}$ curves to be $481.34,356.11$ and $276.89 \mathrm{~F} / \mathrm{g}$ at 10,20 and $50 \mathrm{mV} / \mathrm{s}$ scan rates, respectively. Such high capacitance performance is attributed to the peculiar arrangement of nanorods over flexible carbon cloth as well as presence of underlying substrate which offers high surface area and small ion transportation path [13]. The peak currents increases as the scan rates increased, which suggests that the integrated $\mathrm{WO}_{3} \mathrm{NRs}$ electrode has fast charge transfer and cation diffusion.

Galvanostatic charge-discharge curve of $\mathrm{WO}_{3}$ NRs in Fig.5c. at current density of $20 \mathrm{~mA} / \mathrm{cm}^{2}$ within the voltage range between 0 and $1.8 \mathrm{~V}$ discloses very fast charging and very prolonged discharge time. The specific capacitance value was calculated from the discharge curve which was found to be $465.6 \mathrm{~F} / \mathrm{g}$ at current density $0.5 \mathrm{~A} / \mathrm{g}$.
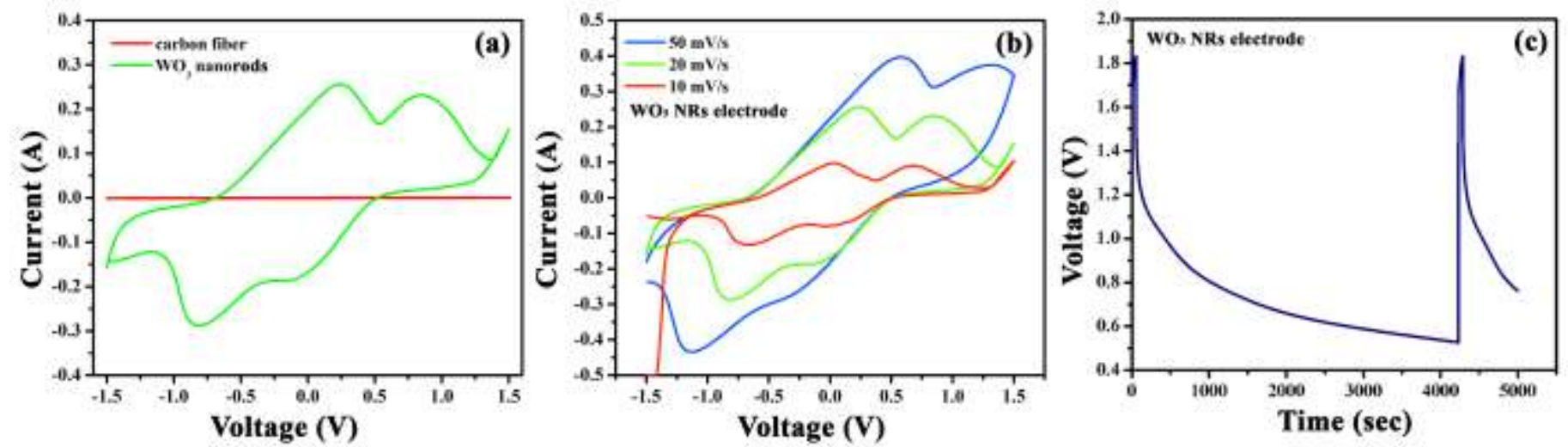

Fig -5: (a) CV curves of carbon cloth and $\mathrm{WO}_{3}$ nanorods electrode at a scan rate of $20 \mathrm{mV} \mathrm{s}^{-1}$, (b) $\mathrm{CV}$ curves of $\mathrm{WO}_{3}$ nanorods electrode at scan rates between 10 and $50 \mathrm{mV} \mathrm{s}^{-1}$ and (c) Galvanostatic charge-discharge curve of $\mathrm{WO}_{3}$ supercapacitor at $0.5 \mathrm{~A} / \mathrm{g}$ current density. 


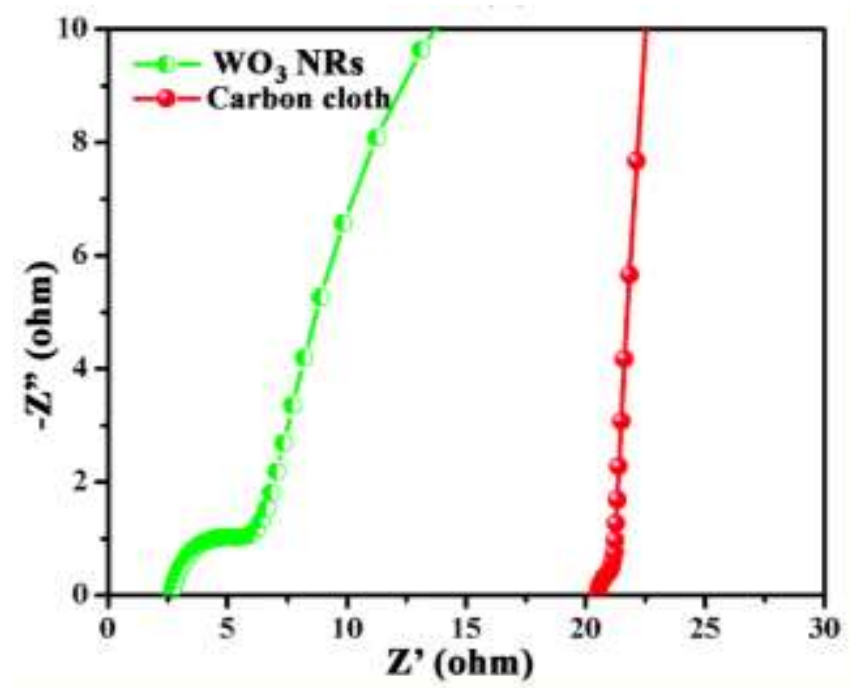

Fig -6: Electrochemical impedance spectra (EIS) of the pure carbon cloth and $\mathrm{WO}_{3}$ nanorods electrode.

Electrochemical impedance spectroscopy (EIS) was carried out to investigate electrical conductivity and to gain more insight of their electrochemical behavior. Fig. 6 shows the Nyquist plot of $\mathrm{WO}_{3} \mathrm{NRs}$ and bare carbon cloth electrodes. The intercept at real $\mathrm{Z}$ axis in high frequency range corresponds to equivalent series resistance $\left(R_{s}\right)$ represents the inherent resistances between the electrolyte and electrode. The intercept of Nyquist curve on the real axis $\left(\mathrm{R}_{\mathrm{s}}\right)$ is $\sim 2.7 \Omega$ for $\mathrm{WO}_{3} \mathrm{NRs}$ whereas the same for bare carbon cloth electrode $\sim 20.56 \Omega$. Such results demonstrate good conductivity and very low internal resistance of the $\mathrm{WO}_{3}$ NRs electrode.

\subsection{Field emission performance}

Generally, nanostructures having beneficiary sharp geometry and high aspect ratio render good field emission (FE) performance where the emission performance is strongly depend on the underlying substrate. Keep all these issues in mind, FE study of the synthesized NRs was executed. Variation of the emission current density (J) with the applied field (E) for these NRs is shown in Fig. 7a. The observed turn on and threshold field values are 3.6 and 8.01 $\mathrm{V} / \mu \mathrm{m}$ respectively where the turn on and threshold fields are defined as macroscopic fields required to produce an emission current density of $10 \mu \mathrm{A} / \mathrm{cm}^{2}$ and $1 \mathrm{~mA} / \mathrm{cm}^{2}$ respectively. The FE characteristics are further analyzed by the Fowler Nordheim (F-N) equation according to which the electron emission process from solid surface can expressed through the equation:

$$
J=\left(\frac{A \beta^{\mathrm{z}} E^{\mathrm{2}}}{\varphi}\right) \exp \left(\frac{-B \varphi^{\mathrm{s} / \mathrm{z}}}{\beta E}\right)
$$

where $\mathrm{A}$ and $\mathrm{B}$ are constants, $\mathrm{J}$ is current density, $\mathrm{E}$ is applied field, $\varphi$ is work function of the emitting materials (5.7 eV for $\mathrm{WO}_{3}$ ) [3] and $\beta$ is the geometrical enhancement factor. The $\beta$ factor enumerates the merit of field emission which strongly depends on several factors like emitter geometry, underlying substrate, conductivity, aspect ratio of nanostructure, inter electrode distance, spatial distribution of emitters etc. [12-14] The FN plot $\left[\ln \left(\frac{I}{E^{2}}\right)\right.$ vs. $\left.\left(\frac{1}{E}\right)\right]$ (inset of

Fig. 7a) exhibits a nice linear relationship in the high field region and suggests that the emission to be of $\mathrm{F}-\mathrm{N}$ type. From the slope of the linear curve, $\beta$ value was estimated which was found to be 4550 .

The excellent $\mathrm{FE}$ characteristics of $\mathrm{WO}_{3} \mathrm{NRs}$ are attributed to their high aspect ratio, the texture. Additionally, woven like geometry of individual strand of fiber provides enhancement the emission performance. The boosting effect can be accounted for by an additional field enhancement factor and thus the overall field enhancement factor of a combined geometry field emitter could be represented as:

$$
\beta_{\text {overall }}=\beta_{\text {nanostructure }} \times \beta_{\text {cloth }} \text {. }
$$

Sharp 1D structures (high $\beta$ nanostructure) and micrometer curvature of the individual carbon strand of the cloth $\left(\beta_{\text {cloth }}\right)$ together give a high field enhancement factor of the sample. Additionally, we have examined the electron emission behavior of the same by varying the electrode separation (Fig. 7b). With the increase anode-emitter distance, the effective emission area increases which ensures greater number of electrons collection at the conical shaped anode and thereby enhancement in over all current density. Such observation of electron emission behavior is consistent with the previous reports. $[15,16]$
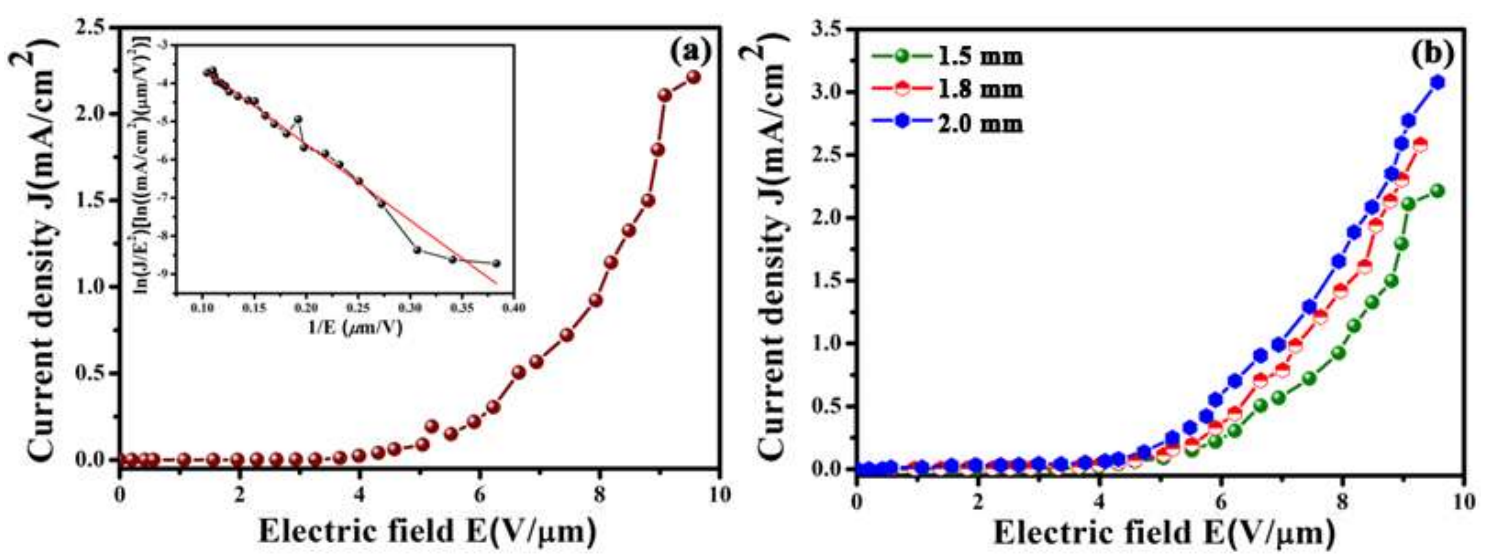

Fig -7: (a) J-E and (inset) $\mathrm{F}-\mathrm{N}$ plots of $\mathrm{WO}_{3}$ nanorods under investigation (b) $\mathrm{J}-\mathrm{E}$ plots of the sample for different vacuum gaps 


\section{CONCLUSIONS}

In a nutshell, we prepared highly crystalline $\mathrm{WO}_{3}$ nanorods over flexible carbon fabric substrate via environmental benign hydrothermal approach. These as synthesized samples should be used for two purposes as they showed excellent electrochemical performance and good electron emission performance as well. By the proper choice of the underlying substrate we bypassed the factors like necessity of binding agent or usage of any ancillary material. Additionally to that the underlying conducting carbon substrate enhances the emission performance also. We believe the NRs over carbon fiber has potential applicability in flexible FE devices namely fully rolled-up field emission display (FED), energy backup system and many more.

\section{ACKNOWLEDGEMENT}

S. Pal thanks the Council for Scientific and Industrial Research (CSIR), Government of India, for providing her a senior research fellowship during the execution of the work. The authors also wish to thank the University Grants Commission (UGC) for financial support under the 'University with Potential for Excellence (UPEII)' scheme.

\section{REFERENCES}

[1] D Shao, M Yu, J Lian and S Sawyer Nanotechnology 24 295701 (2013)

[2] Y Baek and K. Yong J. Phys. Chem. C. 1111213 (2007)

[3] L Li, Y Zhang, X Fang, T Zhai, M Liao, X Sun, Y Koide, Y Bando and D Golberg J. Mater. Chem. 216525 (2011)

[4] L F Zhu, J C She, J Y Luo, S Z Deng, J Chen and N S Xu J. Phys. Chem. C 11415504 (2010)

[5] M Sadakane, K Sasaki, H Kunioku, B Ohtani, R Abe and W Ueda J Mater. Chem. 201811 (2010)

[6] J Wang, E Khoo, P S Lee and J Ma J. Phys. Chem. C 11214306 (2008)

[7] C C Liao, F R Chen and J J Kai Sol. Energy Mater. Sol. Cells 901147 (2006)

[8] S Gubbala, J Thangala and M K Sunkara Sol. Energy Mater. Sol. Cells 91813 (2007)

[9] X P Wang, B Q Yang, H X Zhang and P X Feng Nanoscale Res. Lett. 2405 (2007)

[10] Y Guo, X Quen, N Lu, H Zhao and S Chen, Environ. Sci. Technol. 414422 (2007)

[11] B Yang, P R F Barnes, W Bertranm and V Luca J. Mater. Chem. 172722 (2007)

[12] U N Maiti, S Maiti, N S Das and K K Chattopadhyay, Nanoscale 104135 (2011)

[13] X Lu, T Zhai, X Zhang, Y Shen, LYuan, B Hu, L Gong, J Chen, Y Gao, J Zhou, Y Tong and Z L Wang Adv. Mater. 24938 (2012).

[14] K Cheng, Z Qiao, S Pang, S Wang, X Han, and Z Du Appl. Phys. Express 510580 (2012)

[15] S Pal, S Maiti, U N Maiti, K K Chattopadhyay J. Mater. Chem. C 24005 (2014)

[16] A Khademi, R Azimirad, A A Zavarian, A Z Moshfegh

J. Phys. Chem. C 11319298 (2009)

\section{BIOGRAPHIES}

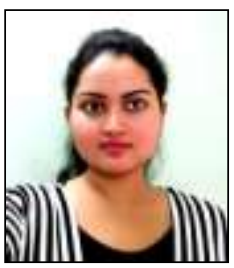

Ms. Shreyasi Pal (born 1989) obtained her B.Sc. (Hons) in Physics from Lady Brabourne College, C.U. in 2010. Later she obtained her M.Sc. in Physics from Calcutta University, Rajabajar Science College in 2012. She has been awarded "CSIR Fellowship" from HRDG, Government of India for academic year 2012-2017. She is now continuing her research as a "Senior Research Fellow of CSIR" at "Jadavpur University".

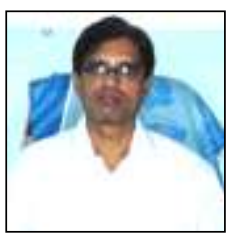

Dr. K.K. Chattopadhyay is a Professor at the Department of Physics, and also the Director of the School of Materials Science and Nanotechnology, Jadavpur University, Kolkata. He has guided so far 24 students for Ph.D. degree and published more than 245 research papers in various international journals of repute, 4 books and a number of book chapters 